Questions de communication

$24 \mid 2013$

Renouvellement des mises en scène télévisuelles de la politique

\title{
Des effets paradoxaux de stratégies de communication : quelques réceptions inattendues d'un magazine territorial
}

About Paradoxical Effects of Communication Strategies: Some Unexpected

Receptions of a Territorial Magazine

Jean-Baptiste Legavre

\section{OpenEdition}

Journals

Édition électronique

URL : http://journals.openedition.org/questionsdecommunication/8706

DOI : 10.4000/questionsdecommunication.8706

ISSN : 2259-8901

\section{Éditeur}

Presses universitaires de Lorraine

Édition imprimée

Date de publication : 31 décembre 2013

Pagination : 199-217

ISBN : 978-2-8143-0182-5

ISSN : 1633-5961

\section{Référence électronique}

Jean-Baptiste Legavre, « Des effets paradoxaux de stratégies de communication : quelques réceptions inattendues d'un magazine territorial », Questions de communication [En ligne], 24 | 2013 , mis en ligne le 01 février 2016, consulté le 02 mai 2019. URL : http://journals.openedition.org/ questionsdecommunication/8706 ; DOI : 10.4000/questionsdecommunication.8706 
JEAN-BAPTISTE LEGAVRE

Centre d'analyse et de recherche interdisciplinaire sur les médias

Université Panthéon-Assas

F-75006

¡blegavre@orange.fr

\title{
DES EFFETS PARADOXAUX DE STRATÉGIES DE COMMUNICATION : QUELQUES RÉCEPTIONS INATTENDUES D'UN MAGAZINE TERRITORIAL
}

\begin{abstract}
Résumé. - L'analyse des réceptions des magazines territoriaux constitue un point aveugle. Une enquête qualitative par entretien auprès de lecteurs d'un magazine d'une communauté d'agglomération de la banlieue parisienne permet d'observer que les stratégies mises en œuvre par les communicants sont susceptibles de réceptions sinon contre-intuitives, paradoxales. À la suite de Robert K. Merton (1936) qui nommait ce type de processus des « conséquences inattendues de l'action », l'enquête permet de questionner des évidences partagées. En effet, le média territorial peut être analysé comme un média reflet, apaisé, dilué. Mais un média tellement reflet qu'il paraît d'abord susciter l'adhésion de ceux qui sont déjà insérés dans le territoire. Un média tellement apaisé qu'il peut être d'abord plébiscité par les récepteurs opposés à l'équipe politique en place. Un média tellement dilué qu'il peut n'être même plus appréhendé comme spécifique et dont les enjeux politiques ne sont plus forcément perçus par les profanes. Autant de constats qui pourraient remettre en cause de nombreuses stratégies de communication territoriale.
\end{abstract}

Mots clés. - Magazine territorial, communicant, réceptions, entretiens, effets paradoxaux. 
e journal territorial a beau être considéré par les communicants' comme le fer de lance de la communication locale (Saurat, Renac, 2009 : I6), il a beau avoir suscité de nombreuses recherches académiques sur ses contenus et sur ses fonctions (Dauvin, 1990 ; Dauvin, Le Bart, I99I ; Leroux, 2000 ; Le Bart, 2000)², ses modes de réception constituent encore un point aveugle. Quelques très rares sondages nationaux ont été réalisés, mais leurs commanditaires - ayant intérêt à penser que le magazine territorial est plébiscité - ne se privent que rarement d'agréger des réponses dissemblables, pour convaincre et se convaincre 3 . Le reste est confidentiel, le plus souvent produit par des agences à la suite de commandes de collectivités, du sondage localisé au focus group très en vogue...

Effectuée à la demande d'une collectivité territoriale, une enquête qualitative par entretiens permet de questionner quelques évidences partagées. Le magazine étudié - un mensuel tiré à 65000 exemplaires proposant à chaque numéro un dossier thématique (l'eau, les transports, le logement, l'emploi, etc.) et un supplément culturel détachable - est celui d'une communauté urbaine, l'une des cinq villes nouvelles d'Île-de-France, dirigée par une majorité politiquement marquée à gauche. Cette communauté regroupe moins de dix communes, au total peuplées de près de 150000 habitants. L'espace est vaste et contrasté. À quelques kilomètres de distance, se côtoient des résidences très privilégiées, des espaces verts étendus et des zones urbaines dites « sensibles », spécifiquement celles d'une ville de 30000 habitants considérée comme « difficile », en pleine rénovation urbaine et disposant encore de plus de $60 \%$ de logements sociaux. L'ensemble du territoire accueille une population plus jeune que la moyenne nationale. Une université se situe juste à côté d'un immense centre commercial et de sièges sociaux d'entreprises.

35 entretiens qualitatifs ont été réalisés, en face à face ou au téléphone, avec une durée variable d'une vingtaine de minutes à 45 minutes ${ }^{4}$. Plusieurs ont été complétés par des échanges de courriels avec les personnes enquêtées ou des appels ultérieurs pour préciser un point resté en suspens. Le mode de constitution

\footnotetext{
| Par le terme « communicants », nous entendons les « dircoms 》, chargés de communication et autres consultants en la matière.

2 L'analyse des producteurs a été délaissée. Pour une exception, voir Philippe Teillet (2000).

${ }^{3}$ On trouve régulièrement des sondages nationaux sur la réception des journaux territoriaux sur le site de l'association qui fédère les communicants publics, en fait territoriaux, Cap'com. Accès : http://capcom.cap-com.org.

${ }^{4}$ Dans l'article, les entretiens réalisés ne sont mobilisés que très partiellement. Ceux-ci étaient couplés (mais non retenus d'une manière explicite dans la présente démonstration) à une centaine d'autres effectués et retranscrits par des étudiants de licence réalisant cette recherche collective dans le cadre d'un séminaire de méthode en sciences sociales. Les résultats de l'enquête étudiante étant stimulants, en 2008 , nous avions proposé à la direction de la communication de la collectivité territoriale concernée de conduire une enquête ad hoc confidentielle, ce qu'elle avait accepté. Nous lui en sommes très reconnaissant et souhaitons la remercier de sa confiance. Merci aussi à Pascal Dauvin d'avoir relu une version antérieure de l'article. Le texte est issu d'une communication à une journée d'étude tenue à l'Institut d'études politiques de Paris (Legavre, 20 I0).
} 
de l'échantillon est dit « en boule de neige », les répondants ayant été présentés par des connaissances habitant l'une des communes du territoire, eux-mêmes pouvant en solliciter d'autres, etc. Un tel échantillon n'interdit pas la diversité - par exemple, un kinésithérapeute appelé par le truchement d'une secrétaire déjà interviewée a pu indiquer deux contacts potentiels, un professeur des écoles et une directrice de communication. Sur les 35 personnes, dix ont été contactées sans intermédiaire et peuvent être considérées comme des relations personnelles. Le trait ne signifie pas un lien fort. Ainsi, parmi d'autres exemples, un restaurateur (présentant luimême plusieurs connaissances) et une serveuse fréquentés de temps à autre le temps d'un repas... Une tentative d'accès en aveugle montre les limites d'une telle stratégie empirique : les refus sont quasiment systématiques. II est difficile de recueillir des propos de non-lecteurs (pourquoi accorder un entretien pour parler d'une non-pratique ?), voire de lecteurs épisodiques du magazine (qu'avoir à dire à un enquêteur sur un média peu familier ?) ou, plus largement, d'imaginer que les motivations sont importantes pour offrir du temps à un enquêteur inconnu, y compris pour un lecteur du magazine mais questionné alors qu'une autre activité l'occupe... La notation n'est pas surprenante : à titre d'illustration, un précédent rapport commandé à une agence par la même collectivité territoriale pour mieux saisir les lectures de ses revues avait nécessité plus de | 200 appels téléphoniques pour recueillir 320 contacts dits « utiles », débouchant sur 95 réponses à un questionnaire et, finalement, seules 18 personnes avaient accepté de se déplacer pour des entretiens de groupe.

Le questionnaire principal était composé de 45 questions très diverses, ne portant pas exclusivement sur le média territorial en tant que tel. Sans doute s'agissait-il de repérer les usages du magazine (temps passé, prises en main, rubriques fréquentées, appréciation de la maquette, désirs de changement, etc.), mais à condition de mieux situer l'enquêté non seulement d'un point de vue sociodémographique, mais aussi dans son environnement territorial (type de logement, type d'insertion territoriale...), médiatique (types de fréquentation du journal municipal, des télévision ou radios locales, des autres médias locaux et nationaux...) ou politique (positionnement sur un axe droite-gauche, participations aux élections nationales et locales, à des associations, etc.).

II serait illusoire d'attendre de chacun plus que des réponses rapides à un questionnaire dont une partie des items était pourtant susceptibles de réponses développées, accompagnées de relances potentielles. Tous les enquêtés n'ont pas les mêmes capacités et envies de verbaliser leurs investissements médiatiques et politiques ou, pour être prudent, « présumés politiques » (Mauger, Fossé-Poliak, 1991), le magazine territorial n'étant pas identiquement considéré comme « politique ». Réponses de bonne volonté, lieux plus ou moins communs, opinions non toujours constituées, volonté de ne pas trop s'engager ou de ne pas perdre la face en coupant court sont loin d'être marginaux lorsque sont abordés des sujets de ce type, spécifiquement dans des milieux dotés de faibles ressources culturelles (Gaxie, 1978). L'enquête n'autorise sans doute pas les conclusions que des 
investigations au long cours en immersion ou par entretiens approfondis rendent possible (spécifiquement Gaxie, 2003 ; Goulet, 2010). Mais, les sens donnés par les agents à leur pratiques et les types de classements qu'ils peuvent effectuer perturbent suffisamment les représentations solidifiées des professionnels pour avoir un intérêt. En effet, l'enquête permet de constater, sur ce cas d'espèce, que les stratégies mises en œuvre par les communicants sont susceptibles de réceptions sinon contre-intuitives, du moins étonnantes, en fait paradoxales. Dans les années 30, le sociologue Robert K. Merton (1936:894) avait désigné d'une heureuse formule ce type de processus : il y voyait des « conséquences inattendues de l'action ». Distribué gratuitement dans les boîtes aux lettres, ce journal territorial peut se lire comme un média reflet, un média apaisé, un média dilué. Un média reflet? Certes, puisqu'il s'agit de mettre en scène le territoire sur lequel vivent les citoyens concernés. Un média apaisé ? Certes aussi, puisque, depuis les années 80 , les stratégies des communicants ont consisté à positiver l'espace local en évitant de le donner à lire comme structuré par des luttes proprement politiques. Un média dilué ? Certes encore, puisque les stratégies des communicants visent le plus souvent à offrir des médias ressemblant le plus possible aux autres médias, des médias « Canada dry » comme peuvent parfois le dire avec distance certains d'entre eux (Legavre, à paraître).

Pourtant, ce type de magazine constitue un média tellement reflet qu'il paraît susciter l'adhésion d'abord de ceux qui sont déjà insérés dans le territoire. Un média tellement apaisé qu'il peut être plébiscité d'abord par les récepteurs opposés à l'équipe politique en place. Un média tellement dilué qu'il peut n'être même plus appréhendé comme spécifique et dont les enjeux politiques ne sont plus forcément perçus par les profanes. En d'autres termes, les écarts et distorsions sont loin d'être anecdotiques entre les désirs des producteurs et les usages que les récepteurs peuvent en faire.

\section{Un média reflet}

À la lecture croisée des entretiens, tout donne à penser que ce ne sont pas, en ellesmêmes, les variables classiques (sexe, âge, diplôme, situation professionnelle...) ou des variables construites pour l'occasion (comme l'exposition aux médias mesurée par le nombre et le type de médias appartenant à l'environnement de l'enquêté) qui expliquent le mieux les comportements des lecteurs étudiés. La variable la plus discriminante paraît être le sentiment d'intégration territoriale. En ce sens, le journal territorial s'apparente à un média miroir, apprécié par ses usagers pour autant qu'ils s'inscrivent positivement dans leur territoire. L'attachement au territoire - et l'attachement hic et nunc - parait être une variable explicative et pas seulement tautologique. La leçon pourrait sembler évidente. Elle est pourtant loin de l'être. 
La première raison est qu'elle va à l'encontre des quelques leçons que les rares chercheurs ayant travaillé sur cette question ont proposées. Ainsi, pour Christian Le Bart (200 : 180), le magazine territorial peut-il procurer une identité de substitution aux individus dominés. II formulerait « une offre identitaire » d'abord susceptible d'appropriation par ceux-là mêmes qui « ne sont pas en situation d'en trouver par ailleurs de plus valorisantes ». Sans doute, cette hypothèse non empiriquement étayée par le chercheur complexifiait l'idée que la plupart se font des stratégies de communication territoriale. En effet, il est souvent admis que ces dernières visent d'abord à produire de l'identité territoriale plus unifiée et unifiante (spécifiquement dans les zones travaillées par de l'intercommunalité ou celles marquées par des clivages ou de la diversité territoriale ou sociale), qu'elles permettent, aussi, de positiver l'espace local de telle sorte qu'une bonne image soit imputable à l'équipe en place lors des élections à venir. En d'autres termes, l'enjeu est de faire parler la ville sous tous ses aspects (et non d'abord proprement politiques, en tout cas partisans), de telle sorte que chacun en vienne à imaginer l'équipe municipale et son maire comme une métonymie valorisante de l'espace local (Le Bart, 1992).

Or, que donnent à entendre les personnes enquêtées? Que le journal territorial fonctionne positivement d'abord pour ceux qui, d'une part, disposent d'autres identités solidifiées et, d'autre part et surtout, pour ceux qui ont des chances d'y adhérer parce qu'ils aiment leur lieu de vie. Le magazine est bien un miroir et ne parait d'abord fonctionner que pour ceux qui s'y regardent avec attrait. Tout se passe comme si les enquêtés ne pouvaient remettre en cause cette équivalence qu'il est possible de formuler comme suit : « J'aime mon territoire, j'aime donc le journal territorial » ou, dans un ordre différent : « J'aime le journal territorial puisque j'aime mon territoire ».

Le constat d'un média miroir pourrait donc susciter questionnement des principaux financeurs. Pourquoi continuer à mettre autant d'énergie et de moyens dans un média qui a des chances de fonctionner, d'abord, pour ceux qui sont prédestinés à l'apprécier ? Cependant, une réponse - double - peut être fournie, même s'il est impossible de la systématiser empiriquement dans la contribution. Rien n'interdit de penser que ces lecteurs qui sont déjà bien inscrits dans le territoire se servent du média comme d'une prise leur permettant de conforter leur bien-être territorial. Mais, le dire suppose d'admettre une ambition beaucoup plus modeste : un média territorial paraît entretenir des identités déjà là plus qu'il ne semble participer à leur émergence. II est aussi possible que, selon un schéma classique, ces lecteurs qui aiment leur cadre urbain puissent faire fonction de leaders d'opinion, offrant à d'autres habitants des modèles de conduite ou des idées en quelque sorte prémâchées qu'ils pourront plus aisément s'approprier - sur le mode classique décrit par Elihu Katz et Paul L. Lazarsfeld (2008 : 199-279). Mais le constat - optimiste - doit être relativisé car, contrairement à d'autres médias, le magazine 
territorial ne parait pas être un objet de sociabilité très poussée ${ }^{5}$. En tout cas, la quasi-totalité des enquêtés déclare ne pas en parler à des tiers, y compris dans la même famille. La question semble même étonner...

Deux entretiens avec des jeunes enquêtés suffiront à se persuader de l'importance d'un ancrage positif dans l'espace local. Les jeunes sont d'autant plus utiles à questionner qu'ils sont souvent considérés comme rétifs à la lecture des journaux territoriaux par ceux qui les conçoivent - de manière significative, et non isolée, les commanditaires de l'enquête avaient pu insister sur le peu d'importance de réaliser des entretiens avec des « jeunes », typifiés comme non-lecteurs, se disant que leurs « cibles » sont plus âgées, spécifiquement dans cet espace urbain où les familles avec jeunes enfants sont nombreuses. Or, tout donne à penser que le fait qu'ils soient jeunes ne dit rien en soi de leur lecture ou non-lecture - pour paraphraser Pierre Bourdieu (1992 : |43-154), la catégorie « jeune lecteur » n'est qu'un « mot ». Ce qui semble expliquer un comportement de lecture est d'abord l'ancrage territorial.

Appelons-le Ali (entretien 19). II vit depuis 199| dans une commune du territoire intercommunal. Ali l'appréhende comme reléguée et hors de l'espace urbain valorisé - une zone pauvre avec des quartiers difficiles, un nombre de logements sociaux très importants, des habitants issus de l'immigration, etc. II confie que ses parents, d'origine étrangère, n'ont pas choisi d'y habiter, alors qu'ils vivaient à Paris, dans le XIX arrondissement et que leur immeuble ancien a été détruit - « Ce n'était pas un choix. On nous l'a imposé [...]. On a refusé, on voulait rester sur Paris. Et finalement, ils nous ont pris de force en nous amenant ici ». Aujourd'hui, Ali est « assistant d'éducation » dans une école d'une commune voisine plus favorisée. II a 25 ans. II dit qu'il se « plai[t] moyennement oui [dans sa commune]. On devient un nom et un prénom à connotation maghrébine... [c'est] pas trop [ça] dans une recherche d'emploi [...]. C'est dans l'inconscient [...]. Là, on est en train de chercher un logement ailleurs ». II a beau avoir vécu l'essentiel de sa vie dans cette ville, il se dit « parisien de cœur » et voit rétrospectivement comme un âge d'or la vie de ses parents à cette époque. « Mais ça ne me gênerait pas du tout [de rester non loin]. Moi, je veux juste quitter [cette ville] [rires] ». II ne lit plus le journal territorial. II l'utilisait quand, lycéen, il devait faire ses devoirs et trouver des exemples à développer ou des exposés - « il y a[avait] une période où je le lisais complètement ». « Je ne m'y intéresse pas trop [aujourd'hui]. Je ne le lis quasiment jamais [...]. Je ne peux pas vous dire si c'est un hebdomadaire, si c'est un bimensuel, un trimestriel, je ne peux pas vous dire ». L'ambivalence ressentie envers l'espace local paraît produire des effets sur sa position de lecteur alors même qu'il dit lire systématiquement des revues que lui prête son voisin de palier, dont Courrier international.

\footnotetext{
${ }^{5}$ Pour des cas inverses de sociabilité poussée, voir Dominique Boullier (2004). Voir aussi l'enquête de Vincent Goulet (2010).
} 
Second exemple : appelons-là Victoire (entretien 35). Elle est étudiante. Elle a 25 ans. Ses parents sont aussi issus de l'immigration. Durant tout l'entretien, elle dit et redit son penchant pour le mensuel, le temps passé à le lire en étant l'un des indicateurs (« 45 minutes, une heure $»)$ :

«- Si le journal territorial n'existait plus, il vous manquerait?

- Ah oui ! Parce que je l'attends, quand même ! Je ne sais pas exactement à quelle période il va arriver mais je sais à peu près à qu'elle période [...]. C'est vrai que quand je rentre, je le vois, je m'assieds et... je me vois même clairement rentrer, pas même enlever mon blouson, être assise sur le coin du canapé et prendre les pages que j'ai l'habitude de prendre... avant d'aller faire ce que j'ai à faire.

- C'est vraiment un rendez-vous que vous attendez...

- Oui »!

Victoire développe aussi son attachement au territoire - et d'autant plus qu'elle a déménagée, qu'elle habite désormais dans le centre de l'espace urbain, juste à côté d'un grand centre commercial autour duquel se sont adossés de nombreux commerces. Socialement, elle n'appartient pas à un monde en tout point opposé à celui d'Ali - il a aussi fait des études supérieures ; le père de Victoire, divorcé, est éboueur; ses parent sont originaires d'Afrique noire.Victoire vivait auparavant dans la même commune qu'Ali. Sa mère est vendeuse au centre commercial. La grande différence est le passé familial sans doute, mais surtout le regard positif qu'elle porte sur son lieu de vie. Et le fait d'avoir quitté la zone urbaine la plus défavorisée pour une autre limitrophe ne fait que renforcer son sentiment d'appartenance à l'espace local. Elle trouve « très très très différents » les deux espaces. Elle est satisfaite d'en avoir changé. Ainsi indique-t-elle ce qu'elle appelle les « pressions » qu'elle ressentait dans son ancienne commune défavorisée pour pouvoir s'habiller librement et qu'elle éprouve encore aujourd'hui lorsqu'elle s'y rend.

«- Le passage d'une commune à l'autre est très marquant. Je vois par exemple vestimentairement, quand je passe... quand j'y vais, il y a certaines tenues que... je sais que je ne m'habillerais pas d'une certaine manière si j'y vais.

- Parce que?

- Parce qu'on sent une pression quand même sociale vis-à-vis des gens, une pression religieuse aussi. Même si ce n'est pas omniprésent, on voit quand même que voilà au niveau de... les critères ne sont pas les mêmes qu'ailleurs.

- Vous vous sentiez très contrôlée?

- Quand même, oui.

- Donc c'était plutôt un soulagement de partir?

- Tout à fait, oui.

- Vous vous plaisez ici?

- Ah oui ! Oui. Carrément. C'est... dans la famille, dans la maison [un appartement], tout le monde est très content d'être ici. Déjà, de par là où on habite, on est à proximité de la gare, notre appartement est très bien placé, on a des bus, le centre commercial est juste en dessous, on est à cinq minutes du grand centre commercial à pied, la gare est à douze minutes à pied, l'université à côté, les écoles, le collège, tout quoi.

- Quand vous étiez dans cette commune [limitrophe] que vous avez quittée, vous aviez l'impression d'être dans la même agglomération?

- Non. Non ». 
Visiblement, Victoire est très attachée au magazine territorial. Elle lit « presque tout », sauf les rubriques qu'elle appelle « politiques » - en fait, l'éditorial et les tribunes politiques ( « Je sais qu'il y a une tribune, il y a trois colonnes là, je ne l'ai jamais lue »). Pour elle, le mensuel n'est pas un magazine politique («Ah non! »). Du coup, le décalage entre les rubriques « politiques » et le reste la frappe :

«- Le [journal territorial], il donne des informations sur tout, sur la vie de [l'agglomération] ce qui se passe, les activités, ce qu'on fait, les sujets d'actualité, etc., et que ces tribunes enfin c'est juste des personnes qui s'expriment sur... ça parait politique alors que le magazine n'est pas politique.

- Vous trouvez que ce n'est pas un magazine politique?

- Ah non! Du tout ! Pour moi pas du tout ».

\section{Un média apaisé}

S'il est une autre caractéristique du média territorial, c'est bien d'être apaisé. Ne pas mettre en scène des conflits. Apporter des réponses plus que soulever des questions. Valoriser l'espace local et ses habitants. En d'autres termes, les communicants se proposent de dépolitiser le message ou, plutôt, d'éviter le plus possible de donner à voir des clivages proprement politiques, en tout cas proprement partisans (c'est-à-dire « politiciens », comme le dit le langage courant). La communauté d'agglomération dont il est question est dirigée par une majorité de gauche, mais des postes de vice-présidents, comme souvent dans pareil cas, sont offerts à l'opposition. En définitive, hors conjoncture électorale ou dossier trop clivant, les acteurs tentent de produire du consensus quand ils ne sont pas sous les regards directs des citoyens. C'est dire que la propension des journaux territoriaux à construire une cité sinon idéale, du moins sans tensions exacerbées est encore plus la norme que d'ordinaire. Comme le disent dans un opuscule deux communicants reproduisant l'idéologie professionnelle partagée : « Le message [...] sera d'autant plus efficace que la publication ne cèdera pas aux lourdeurs de la propagande » (Saurat, Renac, 2009 : 15). Des réceptions pourraient cependant en surprendre plus d'un.

En ce sens, retenir des entretiens de femmes lectrices du journal territorial est suggestif. Pas tant (ou pas d'abord) parce qu'il y aurait un effet de genre univoque - toutes les femmes interviewées n'aiment pas le journal territorial6. Mais, lorsqu'elles sont questionnées, elles sont plus souvent enthousiastes, moins souvent dans la retenue, plus franchement dans l'évaluation positive du média. Faut-il alors parler de différentiel d'affects ? Peut-être si l'on entend par là qu'elles paraissent pouvoir plus aisément accepter de s'approprier le média sans réserve devant un tiers enquêteur. En tout cas, les répondants les plus enthousiastes sont des femmes (sans que la variable socioprofessionnelle ne vienne parasiter le constat). Les lecteurs usant plus facilement de possessifs, d'interjections ou de phrases impératives sont des lectrices.

\footnotetext{
${ }^{6}$ Sur les effets de genre en matière de lecture de presse quotidienne régionale, voir Sylvie Debras (2003).
} 
Elles disent aussi avec plus de véhémence le manque qu'elles éprouveraient s'il venait à disparaitre ou le sentiment positif qu'elles ressentent à chaque livraison?. II en va ainsi avec cette secrétaire (entretien 14) qui garde la collection depuis le premier numéro et qui nomme son magazine territorial « le journal » :

«- Le [journal territorial], vous le lisez à chaque fois?

- Oui, oui !

- C'est un rendez-vous?

- Oui, oui ! Je le trouve dans ma boîte aux lettres et quand je ne l'ai pas, je n'aime pas trop. D'ailleurs une fois, j'ai téléphoné à la maire : "Je n'ai pas eu le journal ! parce que la mairie [...]". - Et, vous en discutez avec des proches?

- Oui, X [sa collègue de bureau]. On discute pas mal, oui.

- "Oui, j'ai vu cet article"...

- Oui, oui... [imitant sa collègue] : "Tiens ça y est ! J'ai eu le journal dans la boîte aux lettres". Alors évidemment, moi je suis toujours la deuxième, alors je dis :"Bon, eh bien tant pis !"».

L'exemple des femmes retenues est intéressant pour saisir que le média ne suscite pas rejet de la part d'électeurs de droite. Les enquêtés qui se donnent à voir comme les soutiens les plus forts du journal territorial se situent d'ailleurs le plus souvent, dans l'échantillon, sur la droite de l'échiquier politique. Cette même enquêtée (entretien 14) dit aimer feuilleter les livraisons rétrospectivement pour voir les fortes transformations de l'espace urbain.

«-Dès que je le reçois je le lis tout le temps... ah oui, oui, oui, ça oui ! Pas tous les articles quand je le reçois... dès fois je le mets de côté pendant 15 jours mais je vais le lire... ça c'est sûr. - Vous y tenez à votre journal!

- Ah oui, oui, oui ! ».

Cette enquêtée habite sa commune depuis plus de 25 ans. D'ailleurs, elle se définit par sa commune plus que par l'agglomération. Elle a aussi un discours très négatif sur la gauche (responsable, veut-elle croire, de la construction d'espaces urbains « très laids », quand la droite aurait l'exclusivité du « bon goût »). La gauche locale majoritaire au niveau de l'agglomération parait être comme « mise entre parenthèses », même si elle vote pour la droite aux échelons local comme national. Elle ne trouve pas le journal territorial orienté politiquement. « Non, non, moi je trouve que c'est objectif »».

Comment une électrice de droite, habitant une ville votant majoritairement à droite, peut-elle être aussi attachée à son média d'agglomération, le trouver « objectif » - assure-t-elle - et aucunement l'aborder comme un vecteur de communication politique d'une communauté d'agglomération dont la majorité

\footnotetext{
7 Cependant, il est possible qu'un effet de genre spécifique se repère, les hommes questionnés ayant plus souvent tendance à dire, lorsqu'ils sont en couple, que le journal territorial est d'abord lu par leurs femmes, sachant que plusieurs femmes disent sélectionner les sorties culturelles du couple ou de la famille à partir d'une lecture du média. L'opposition entre le « dedans » et le « dehors » analysé par P. Bourdieu (1998) dans La Domination masculine se rejouerait d'une autre manière : non plus l'espace public à l'homme et l'espace privé à la femme mais, en quelque sorte, l'espace local à la femme et sous une forme non proprement politique. Voir aussi l'enquête de $V$. Goulet (20 I0) qui va dans le même sens lorsqu'elle analyse les usages des médias par les classes populaires.
} 
est marquée à gauche ? En fait, une piste étayée par plusieurs entretiens (spécifiquement cinq entretiens : 4, 8, 14, 22 et 23) peut en fournir plus qu'un début d'explication. En entretien, ces femmes disent rejeter la politique - trois votent systématiquement, mais l'une ne vote qu'occasionnellement. Elles mettent aussi à distance les médias d'information politique et générale. Ils seraient des messagers de mauvaises nouvelles. C'est ce qu'avance notamment cette enquêtée qui, en conséquence, préfère lire le journal territorial :

«- Elles ne vous attirent pas tellement en ce moment les infos nationales...

- Eh bien non parce que... comme beaucoup de gens diraient que ce n'est que du négatif et moi le négatif...

- Ah oui, vous trouvez qu'elles sont toujours négatives?

- Oui... alors que je suis sûre qu'il y a beaucoup de choses positives mais on n'en fait pas cas. Alors moi, je préfère lire le journal territorial et même le relire...

- Parce que ça ne vous semble pas toujours une façon négative de voir...

- Mais au contraire c'est positif moi je trouve parce qu'il y a beaucoup de choses et on en fait de plus en plus et on ne laisse personne à l'écart. II peut très bien y avoir un reportage sur le troisième âge, cinq pages sur le troisième âge, enfin j'exagère peut-être en disant cinq, mais bon tout le monde est concerné...

- Mais vous trouvez qu'à la télévision ou ailleurs, les médias sont trop négatifs?

- Oui. Ah oui, alors là oui ! Je suis catégorique. C'est négatif.

- ça vous déplaît...

- Oui... je crois que cela a un impact sur... enfin, c'est stressant. Les mauvaises nouvelles sont stressantes! Comme il y en a beaucoup de mauvaises nouvelles, je ne dis pas qu'il ne faut pas se tenir au courant de ce qui se passe, ce n'est pas la question... mais moi je peux très bien aussi acheter un autre magazine à l'occasion... je ne sais pas, même un magazine télé comme Télérama, pourquoi pas.

-Vous lisez des magazines ou des journaux...?

- Alors... ça m'arrive d'acheter un Paris Match ou un truc comme ça, s'il y a un événement qui s'est produit ou qui m'intéresse.

- C'est fréquent?

- Non... ».

Une retraitée qui continue de travailler en gardant des enfants après l'école adopte un discours assez proche (entretien 4). Elle ne lit aucun média en dehors de ses deux magazines territoriaux (celui de la commune et celui de la communauté d'agglomération) qu'elle regarde dès qu'elle les reçoit. Elle paraît avoir de plus en plus de mal à regarder aussi les informations télévisées qu'elle consomme une fois par jour:

« C'est trop triste, on ne voit que des mauvaises nouvelles, c'est vrai ! Un peu de gaîté franchement, cela ne ferait pas de mal... ils n'annoncent que des mauvaises nouvelles et moi cela ne m'intéresse pas ».

Mais ce n'est pas tout. Si non seulement son appréciation du média territorial paraît être en lien étroit avec son rejet des autres médias qui apportent des « mauvaises nouvelles », c'est aussi qu'elle n'aime pas la politique abordée comme un espace de «mensonges » profitant à ceux qui occupent les « places ». Elle ne lit d'ailleurs pas les tribunes politiques et rarement les éditoriaux publiés par le mensuel territorial : 
«- Pour moi, ils [les hommes politiques] se valent tous, c'est toujours pour les mêmes, c'est...

-Vous n'aimez pas la politique...

- Pas du tout parce qu'il n'y a aucune aide pour nous... il n'y a vraiment rien [...] que ce soit la droite ou la gauche, on est au même stade » (dit cette femme qui précisera à la fin de l'entretien qu'elle vote en fait à droite).

En conséquence, en offrant une vision de l'espace local sans conflits, le mensuel pourrait susciter une adhésion plus « évidente » à des agents sociaux moins adeptes de conflits et se positionnant à droite de l'espace politique. Loin de la politique politicienne qui divise. Le mensuel leur convient d'autant plus qu'il se positionne à travers, d'abord, ce que les Anglo-saxons appellent des soft news, en tout cas un traitement de l'information qui n'appréhende pas le monde au prisme de ses dysfonctionnements et autres discontinuités du monde social telle la presse dite de « qualité » l'envisage le plus souvent (les hards news) ${ }^{8}$.

Le cas d'une autre femme secrétaire plus jeune d'une bonne quinzaine d'années renforce cette appréciation (entretien 23). Elle appartient aux milieux sociaux dominants, moins par ses diplômes, activité professionnelle (bien qu'elle ait auparavant été « responsable d'une boutique » dans la « gastronomie de luxe ») ou le lieu d'exercice de sa fonction professionnelle, que par son mariage et son lieu d'habitation. Son mari est cadre supérieur et elle qualifie son milieu social de « très bien, un milieu de quand même favorisé, il ne faut pas se voiler la face. Maintenant $[. .$.$] ce n'est pas parce qu'on habite dans un quartier un peu favorisé$ que l'on oublie les autres $\gg$. Le couple est propriétaire d'une maison individuelle dans un des quartiers les plus réputés de l'espace territorial. Elle est visiblement peu à l'aise avec la politique, ses modes de classements et les potentiels conflits qu'elle peut susciter - à plusieurs reprises, cette femme exprime sa gêne au cours de l'entretien soit par le rire contraint, soit en avançant : « C'est vraiment très indiscret. Oui, c'est très indiscret [ces questions posées]. [...] Alors... c'est vrai, la politique c'est un sujet... presque tabou entre guillemets [rires] ». Elle est particulièrement gênée lorsqu'il lui est demandé de s'autopositionner sur un axe droite-gauche, comme de situer socialement son milieu d'origine autant qu'actuel. Elle se place « plus [à] droite effectivement ». Cette secrétaire finit par répondre à la question : «Vous le trouvez orienté (le journal territorial) ? », « Je vais vous dire. Je me sens détachée de la politique, parce que peut-être suite à des déceptions [elle se situe à droite, mais n'apprécie pas en tout point Nicolas Sarkozy, président de la République au moment de l'enquête]... mais c'est vrai qu'il est quand même orienté [vers la] droite ».

\footnotetext{
${ }^{8}$ || pourrait être objecté que le magazine a d'autant plus de chance d'être consensuel que les communautés d'agglomération sont souvent gérées d'une manière moins conflictuelle que les communes où les clivages droite-gauche sont plus visibles et très souvent réactivés par les élus, au moins dans les grandes villes. Mais, d'une part, la communauté d'agglomération étudiée est celle d'une ancienne ville nouvelle disposant historiquement de pouvoirs propres beaucoup plus importants du fait d'une législation spécifique et, d'autre part, le magazine en tant que tel n'est guère différent dans ses formes et contenus que celui d'un magazine communal ordinaire.
} 


\section{Un média dilué}

Un média de communication appréhendé comme un média ordinaire par les profanes produit-il les effets escomptés par ses producteurs? Le plus optimiste des communicants rétorquera que l'objectif sera précisément atteint si le média territorial se fond dans le paysage médiatique. Le média donnera une bonne image de l'espace local ; à terme, l'équipe politique en tirerait des soutiens. Le sociologue plus pessimiste (ou réaliste ?) n'hésitera pas à poser une question subsidiaire : mais qu'en est-il si le média n'est même plus guère appréhendé, à tout le moins par une fraction de récepteurs, comme un média de l'institution qui le finance? En d'autres termes, il se pourrait que les producteurs de médias de communication soient dépassés par leurs propres créatures, le média territorial étant perçu de facto comme un média territorial parmi d'autres, et comme un média gratuit parmi d'autres et, plus largement, comme un média parmi d'autres médias. C'est que la boîte aux lettres de chaque citoyen est aujourd'hui peuplée de nombreux magazines territoriaux (de la commune à l'agglomération, au département, voire de la région...) sans compter que beaucoup d'urbains disposent de journaux gratuits dans les gares, métros et autres lieux publics. En plus de la presse quotidienne régionale - et, spécifiquement, Le Parisien, très lu dans ce département de l'Île-de-France -, ils apportent, eux aussi, des informations locales. Plus largement, les récepteurs des magazines territoriaux peuvent aussi lire d'autres médias...

Le journal territorial est bien un outil de communication : le constat réaliste est, en même temps, trivial. Cependant, il est utile de le rappeler : les médias d'organisation (magazine de ville, magazine d'entreprise, lettres de donateurs d'associations caritatives, etc.) ont tendance à mimer pour mieux masquer ou, à tout le moins, pour mieux s'insérer. La stratégie des communicants vise à adopter les codes de la presse ordinaire - visuels, « chemin de fer », mise en page, etc. « À la manière de... », conseillent les experts ${ }^{9}$. II s'agit bien de fondre le média territorial dans l'ensemble des médias de sorte qu'il suscite une adhésion perçue comme plus naturelle. Parce que les communicants considèrent que la politique a toutes les chances d'être vue comme politicienne par les lecteurs potentiels, ils tentent de transformer le journal territorial en « magazine de ville », comme ils les nomment parfois (l'ancien « bulletin municipal » sera symétriquement perçu comme archaïque et le magazine comme un indicateur de « professionnalisation »). Le projet consiste à accorder une place sans doute importante aux politiques publiques - mais sous un angle concret, dépris de clivages proprement politiques et en tant qu'elles produisent des effets pratiques sur les citoyens. II vise aussi, et parfois d'abord, à mettre en scène des habitants typiques ou originaux et les activités culturelles de l'espace local (par exemple, un guide culturel est inséré

\footnotetext{
9 Même si T. Saurat et L. Renac (2009 : 47) peuvent ne pas oublier l'objectif qu'ils qualifient d'hybrides, « il [le journaliste territorial] écrit à la manière de, pour une publication à la manière de, mais avec un objectif à la fois informatif et communicant ».
} 
dans le magazine territorial), en d'autres termes à positiver l'espace local pour escompter une prochaine imputation à l'équipe municipale, rendre compte des activités associatives et culturelles qui se tiennent au niveau local pour donner à penser que le maire en rend possible l'épanouissement...

En conséquence, les luttes politiques sont cantonnées aux tribunes politiques, rendues obligatoires par la loi qui impose de donner la parole aux groupes politiques présents dans les conseils. De même, lorsque les communicants sont en position de le faire ou lorsque le maire ou président de Conseil est sensibilisé à la question, si l'éditorial n'est pas dépolitisé, du moins ses aspérités sont le plus possible gommées. D'ailleurs, marginaux sont les lecteurs questionnés qui déclarent lire les tribunes politiques reléguées en fin de magazine. Minoritaires sont ceux qui disent lire les éditoriaux - certains n'ont même pas conscience, y compris parmi les lecteurs les plus attentifs au média, y compris parmi les lecteurs usagers de médias écrits ordinaires, de l'existence d'un éditorial signé par le premier édile local...

II arrive aux lecteurs d'identifier spontanément l'émetteur, la communauté d'agglomération (d'ailleurs, ils peuvent avoir du mal à identifier son signataire est-il maire? quel est son nom ?...). Et le constat ne touche pas que les catégories populaires. En témoigne un universitaire (entretien 2I) et ce, même s'il ne l'exprime pas avec les mêmes mots et de la même façon que d'autres enquêtés. I| habite depuis huit ans une des grandes communes de l'agglomération. II se positionne au centre droit de l'espace politique. II vient d'avancer qu'il ne trouvait « pas du tout, pas du tout », le magazine engagé politiquement.

\footnotetext{
«- Les pages politiques, tu ne les lis pas?

- Je n'y fais pas attention. J'avoue que là ça me passe au-dessus de la tête. C'est vrai que moi, je le perçois plus comme un magazine d'informations pratiques. Et c'est vrai que sur les débats éventuels de fond, ça me passe vraiment au-dessus de la tête. Mais je reconnais aussi que j'ai du mal à m'impliquer concrètement et même émotionnellement dans ce qui se passe ici.

- Et l'éditorial du président de la communauté de l'agglomération?

- Par accident à un moment où j'ai vraiment le temps et où je décide, mais ce n'est pas fréquent, de le lire de $A$ à $Z$... et là je peux commencer par l'éditorial. Mais tout dépend du type de l'éditorial. Là, dans le dernier [il le prend en main], "un territoire culturel riche" [rires], ça n'a pas retenu mon attention et spontanément je suis passé à la suite...

- Tu ne cites pas le nom du signataire, tu ne connais pas le nom du président de l'Agglomération? - Je rigole toujours à la télé quand ils posent des questions sur les élus et que les gens sont incapables de répondre et c'est bien de rire... mais quand on est concerné [rires]... Joker ! Je ne sais plus »!
}

Comme beaucoup d'autres, cet enquêté ne perçoit pas le magazine territorial comme délivrant un message politique mais comme « un magazine d'informations pratiques », une sorte « d'outil de veille » des activités au local, comme il le dit plus loin dans l'échange. D'ailleurs, imaginer des agents sociaux maitrisant tous d'une égale manière les distinctions entre « information » et « communication » serait une erreur. Le mot même de « communication », voire de « propagande » ou autre « démarche commerciale », n'est retenu que par une petite fraction d'enquêtés - composée de deux populations plutôt rétives au média territorial, y compris quand ils en sont politiquement proches, les militants politiques (dont ceux se 
situant à gauche) et ceux qu'il serait possible d'appeler les « artistes » (c'est-à-dire ayant un engagement artistique à titre professionnel principal ou secondaire, passé ou présent). Mais, la plupart des enquêtés ne paraissent pas se poser la question d'une lecture potentielle à l'aune de cette opposition structurante - au moins dans l'espace des initiés (Legavre, 20l I) - entre journalisme et communication. En définitive, ils ne semblent pas appréhender le média territorial comme un média de communication. La notation n'est pas aussi surprenante qu'il y parait. Du moins, une équipe de chercheurs (Croissant et al., 2005) avait fait le même constat avec un média de communication, le magazine culturel de la FNAC, Epok. Réalisant une enquête de réceptions auprès de lecteurs de ce magazine de marque, elle notait l'« intériorisation d'une vision très vaste et corollairement peu discriminante de "l'information" ». Et d'ajouter : « Que celle-ci puisse avoir une finalité promotionnelle (comme dans la communication d'entreprise), ou non (comme dans le journalisme), a finalement peu d'importance pour les lecteurs. Leur conception de "l'information" est beaucoup plus large ». De telle sorte que les usagers de ce magazine culturel tendaient à « se plac[er] dans leur grande majorité en surplomb de cette opposition » (Croissant et al., 2005 : 82-84).

Puisque les notions d'information et de communication n'ont guère de sens pour la plupart des enquêtés, c'est autour de l'engagement du média, de son orientation politique que des questions ont été posées («Est-ce que vous trouvez que [nom du journal territorial] est engagé, orienté politiquement? »). Typique de ce point de vue, un homme travaillant comme ingénieur-qualité (entretien 10) - se classant lui-même à droite - avance : « Je ne sais pas dire parce que la politique ne m'intéresse pas non plus donc même si c'était marqué politiquement de par les propos, peut-être que je ne ferais pas l'analyse de dire :"Tiens, on voit transparaître tel ou tel mouvement politique" ». De même, une femme médecin (entretien 8), qui se positionne aussi à droite, avance spontanément : « Je trouve que c'est neutre politiquement. Peut-être que je me plante, mais moi je trouve que c'est le cas. Comme ce n'est pas du tout ce que je recherche [la politique] dans ce journal-là... »».

Cette impossibilité à verbaliser ou à s'appuyer sur des classements communs aux producteurs de supports d'information ou de communication touche - contre toute attente - l'ensemble des catégories de lecteurs ${ }^{10}$. Y compris quand les enquêtés lisent des médias d'information qui pourraient les sensibiliser à produire de la différence et appliquer les classements ordinaires à ses producteurs. On peut lire Libération ou Le Nouvel Observateur et ne pas distinguer le média territorial en tant qu'il serait, contrairement aux autres médias fréquentés, un média visant à promouvoir la communauté d'agglomération, son territoire et l'action de ses élus. On peut lire Télérama, Le Parisien, Le Figaro ou Marianne et attendre son journal territorial, et y marquer un attachement. D'ailleurs, deux enquêtés (entretiens 8 et 14) considèrent le média visé spontanément comme un journal de la presse

10 Cette fois, le résultat est inverse de celui de l'étude portant sur Epok (Croissant et al., 2005) puisque ses auteurs observaient que les lecteurs disposant de ressources culturelles n'adoptaient pas en tout point le même discours que les autres enquêtés. 
quotidienne régionale. Par un apparent paradoxe, le trait paraît d'autant saillant que les enquêtés résistent aux messages proprement politiques. En d'autres termes, les messages explicitement politiques, incarnés dans le média territorial par des élus identifiables ne sont pas forcément vus, en tout cas retenus comme dignes d'intérêt par tous les lecteurs. Comme l'écrit le sociologue Richard Hoggart (1957 : 296) dans un tout autre contexte, « ils ne lisent [...] que d'un œil ».

Ce constat pourrait faire penser qu'un concept à succès, l'attention dite oblique, ou le « regard oblique » (ibid.), rend bien compte de catégories de pratiques ou, en tout cas, du discours sur ces pratiques. Mieux, il semble devoir dépasser le seul cas des groupes socialement dominés. Le concept, proposé par Richard Hoggart dans un livre déjà ancien est devenu classique. La Culture du pauvre visait à montrer que les classes populaires étaient loin d'être passives à l'égard des messages émis, par la publicité ou, plus largement, par ceux qui souhaiteraient les « conditionner » à travers « la presse populaire », « la presse de grande diffusion » ou « à scandale » (l'enquête se situe au Royaume-Uni), pour reprendre les termes datés de l'auteur. Pour le sociologue, les membres des classes populaires produisent ainsi une forme de « résistance », pas forcément consciente et maîtrisée, contribuant à « neutraliser les aspects les plus virulents du message ». Richard Hoggart (ibid. : 296-295) retient aussi une autre expression : une « consommation nonchalante ».

Pourtant, la situation parait ici plus complexe. D'une part, les lecteurs questionnés semblent certes « résister » à l'emprise politique ; d'autre part, ils paraissent le plus souvent ne pas non plus maîtriser le positionnement latent du vecteur de communication. En définitive, ils mettent à distance la politique ordinaire - des prises de positions clivées, des signes extérieurs d'existence (des élus éventuellement identifiables sur des photos ou ayant des titres à parler). Mais ils ne voient pas forcément le reste... II y aurait, en quelque sorte, la politique politicienne - celle des luttes politiques ou celles d'élus prenant la parole en nom propre et essentiellement appréhendées par l'éditorial et les tribunes politiques, du moins quand ils sont « vus » comme tels - et « tout » le reste du magazine qui ne parait pas immédiatement pensé par les lecteurs comme politique. Du coup, pour eux, il n'y aurait guère de différence entre le journal territorial et les autres médias d'information politique et générale. Ou, plus précisément, les lecteurs questionnés peuvent produire des différences entre vecteurs de diffusion, mais pas là où il pourrait être attendu...

Pour s'en persuader, et après quelques entretiens, il avait été décidé que, lorsque la personne interviewée indiquait qu'elle lisait d'autres médias d'information politique et générale, nous lui demanderions systématiquement de les comparer au magazine territorial. L'exercice est artificiel - les enquêtés ne le font pas d'eux-mêmes -, mais il a le mérite de montrer que les rationalisations produites ne se fixent que rarement sur les catégories des experts (communicants, journalistes, chercheurs...). Certes, une petite fraction des lecteurs distingue une presse qu'elle va alors qualifier de « presse institutionnelle » ou de « magazine institutionnel ». Mais ces répondants sont très typés : ils sont élu, ex-militant, communicant, universitaire, cadre de l'administration de la communauté d'agglomération... Pour les plus nombreux - d'appartenances sociales 
très diverses -, deux options sont repérables. La première leur permet de classer tous les médias comme étant « engagés », le journal territorial ne faisant pas exception. La « communication » serait alors une forme d'engagement parmi d'autres. Elle ne nuit donc pas, en elle-même, à la lecture de chacun. Cette position très minoritaire, en tout cas très rarement verbalisée comme telle, est retenue par un kinésithérapeute (entretien 24). II dit lire deux quotidiens par jour et au moins un hebdomadaire par semaine. II se positionne « plutôt à droite », « mais c'est très complexe, surtout en ce moment », ajoute-t-il. I| lui est d'abord demandé s'il trouve que le journal territorial « est engagé du point de vue politique ». II répond par la négative : « Je n'ai pas cette impression ». II précise seulement qu'il ressent dans le traitement de l'information une rivalité entre les deux grandes villes de l'agglomération - dont la ville qu'il habite et dirigée par une équipe du centre droit, contrairement à la plupart des autres communes de l'agglomération. Lorsqu'il lui est demandé s'il peut en être gêné, il répond : « Non. Cela ne me gêne pas parce qu'au niveau national, il n'y a pas un seul journal qui n'est pas engagé ». Ensuite, l'homme ajoute qu'il lit « peu, [en fait] non ['éditorial du média territorial] » et pas plus les tribunes politiques («Non plus »). Seconde solution retenue plus souvent par les enquêtés, sans qu'il y ait apparemment un lien évident avec le milieu socioprofessionnel ou les ressources culturelles : ils proposent des distinctions entre types de médias fondés non sur la nature de l'émetteur ou du message, mais sur la zone couverte, la fréquence de parution ou les thématiques retenues. Dans cette optique, Libération est différent du média territorial, mais parce qu'il est quotidien et traite de l'actualité nationale et internationale. Le Parisien est différent du média territorial mais parce qu'il est quotidien, qu'il traite de l'Île-de-France et qu'il se focalise sur les faits divers, etc. Plusieurs illustrations peuvent être retenues, cette prise de position n'étant pas corrélée (en tout cas dans l'échantillon retenu) à la détention de ressources culturelles ou sociales. Elle peut prendre des formes distinctes : quatre peuvent être présentées.

Ainsi une femme, commerciale, de 44 ans (entretien 13) habite-t-elle depuis 30 ans dans la plus grande commune de l'agglomération. Ses parents y vivaient déjà quand elle était enfant. Elle a fait une « petite » école de commerce et occupe une position plutôt basse dans l'espace de sa profession. Elle est mariée à un chauffeur de taxi. Elle est issue d'un milieu ouvrier et continue d'ailleurs encore aujourd'hui de se qualifier d'《 ouvrière », même si ses enfants indique-telle n'aiment pas ce terme - « Moi, par rapport à mes parents, je reste ouvrière ». Elle lit Libération tous les jours (elle l'achète, dit y être « très attachée »; elle le lit depuis 15 ans) et parcourt Le Parisien (« au travail ») irrégulièrement, elle achète Elle et des magazines de décoration de temps à autres. Elle donne à voir à l'enquêteur des signes extérieurs de politisation. Elle se positionne à gauche (« Je suis de gauche, moi! »). Elle se dit nostalgique de mai 68 qu'elle n'a pas vécu (« J'aurais aimé connaître mai 68 [...], je trouve que les gens de ce temps-là avaient plus de poigne, ils savaient ce qu'ils voulaient alors que maintenant les gens [...] sont plus individualistes, chacun pour soi »). Elle lit le magazine territorial, mais sans grande intensité. Elle répond par la négative quand il lui est demandé si elle le trouve « marqué politiquement ». « Non, non », indique-t-elle. Elle ne lit jamais l'éditorial, ni les tribunes politiques. 
«- Quand vous comparez Libération et [le magazine territorial], vous vous dites quoi?

- Je trouve que ce n'est pas comparable.

- Pourquoi?

- Parce que Libé c'est ce qui se passe dans le monde, politiquement, alors que [le magazine territorial] c'est local. On ne peut pas comparer les deux.

- Pour vous la différence, c'est moins dans le traitement de l'information que dans la zone couverte?

- Voilà ».

Deuxième illustration : l'entretien effectué auprès d'un cadre supérieur de France Telecom (entretien 16). L'homme est autodidacte et a passé des concours internes lui permettant de progresser dans la hiérarchie. II a 47 ans, il est gros consommateur de médias, en particulier écrits (surtout des magazines) et lit souvent Le Parisien.

«- Si vous aviez à comparer Le Parisien et [le magazine territorial], qu'est ce que vous diriez ?

- Rien! lls ne sont pas comparables.

- Pourquoi ?

- Ce n'est pas la même approche. Le Parisien, ça reste un journal d'informations générales, même s'il y a des articles sur la région ; ça reste général. [Le magazine territorial] est plus profond, au sens où l'on rentre un peu plus dans le cœur de la ville.

- Donc c'est plus une question d'espace géographique pour...

- Oui.

- Dans le traitement de l'information, ça ne vous semble pas foncièrement différent?

- Je n'ai pas forcément été attentif pour répondre de manière... Non, je ne sais pas. Je ne peux pas vous répondre là-dessus. À la limite... pour moi, c'est différent. Et donc à partir du moment où je me suis mis dans mon esprit que c'est différent je ne vais pas forcément chercher à les comparer ».

Troisième exemple, l'entretien réalisé avec une jeune retraitée de 55 ans (entretien 26). Cette ancienne cadre à La Poste est aussi abonnée à Marianne, Télérama, Le Parisien. Son mari achète Le Canard enchaîné. Elle dit se procurer tous les 15 jours Le Monde. Là encore, et même si elle distingue pour une part un mode de traitement (Le Parisien traite des faits divers), elle ne s'appuie pas sur la nature du média - un journal de communication - pour expliquer ce qui lui semble aller de soi : «Ce n'est pas pareil ». Le Parisien est quotidien, il parle des « chiens écrasés », propose des articles nationaux, il n'a en commun avec le média territorial que certains thèmes (des résultats sportifs et une actualité économique localisée).

«- Si vous aviez à comparer Le Parisien et [le magazine territorial] ?

- Ce n'est pas pareil quand même... ah non!

- Pourquoi?

- Le Parisien, il y a quand même... d'abord c'est national, il y a quand même des articles nationaux.Après quand on prend sur les communes du coin, c'est quand même, la rubrique des chiens écrasés. Peut-être quand même le côté sportif... on voit que [cite une commune] a gagné au foot contre [une autre commune du département] comme la semaine dernière. On va le savoir sur [le journal territorial] peutêtre. Il y a certaines rubriques que l'on va retrouver mais je trouve que ce n'est pas du tout pareil quand même. Après, peut-être dans Le Parisien-économie, on va retrouver des choses du [journal territorial] sur des thèmes spécifiques. Et puis i l y en a un qui est quotidien et donne des nouvelles au jour le jour ».

Le dernier exemple proposé pour indiquer la variété des prises argumentatives des enquêtés sur ce point est offert par un enseignant (en sciences) de collège (entretien 24), ayant la cinquantaine. II vient de préciser que Le Parisien, c'est « mon journal ». La différence est cette fois-ci la périodicité : 
«Si vous aviez à comparer [le magazine territorial] et Le Parisien?

- Ce n'est pas du tout pareil. Il y en a un, un quotidien qui traite des faits divers, l'autre c'est plutôt pour l'information de la ville.

- Pour vous, la différence, ça serait le territoire traité?

- Oui, oui. Je ne sais pas... [le magazine territorial], il sort tous les mois, non?

- Oui.

- Donc ça n'est pas pareil »!

\section{Conclusion}

Des initiés pourront trouver à bon droit que ce qui précède n'est pas si surprenant. En effet, nombreux sont les travaux en sciences sociales qui rappellent que « l'étude des réceptions enseigne à accepter le désordre du présent et du quotidien, à résister à la tentation d'une explication unique et "évidente" » (Le Grignou, 2003 : 213 ; Rieffel, 2005). N'y a-t-il rien d'étonnant alors que certains croient construire un média producteur d'identités quand il entretient pour beaucoup du déjà là, faisant fonction de média miroir? N'y a-t-il rien d'insolite si le magazine, tellement « apaisé », semble plus aisément approprié et plébiscité par une fraction de lecteurs (de droite) ayant peu de chance de soutenir les commanditaires (de gauche) ? N'y a-t-il rien d'inattendu si ce média dilué peut ne même plus être perçu pour ce qu'il est, soit un média produit justement par des acteurs politiquement situés dans l'espace local ? Peut-être. De fait, le média magazine territorial s'est installé comme une évidence parmi les outils des communicants, de telle sorte qu'il constitue désormais un « genre » peu questionné au sein duquel les écarts de contenus ou de maquettes paraissent finalement assez faibles, chacun semblant persuadé que ses propres stratégies de communication imprimée sont sinon efficaces, du moins conformes. Mais, après tout, comme le soulignait fortement Robert K. Merton (1936: 898, 90I) constatant que les mauvaises anticipations ont le plus souvent pour cause un défaut de connaissance, les acteurs tendent à se focaliser sur les effets immédiats des décisions prises, en excluant d'autres conséquences plus complexes à appréhender et souvent plus tardives...

\section{Références}

Boullier D., 2004, La télévision telle qu'on la parle. Trois études ethnométhodologiques, Paris, Éd. L'Harmattan.

Bourdieu P., 1992, « La “jeunesse” n'est qu'un mot », pp. I 43- I 54, in : Bourdieu P., Questions de sociologie, Paris, Éd. de Minuit.

— 1998, La domination masculine, Paris, Éd. Le Seuil.

Croissant V., Rebillard F., Spano W., Touboul A.-L., 2005, « De la culture comme image de marque aux marques journalistiques: Epok, le consumer magazine de la FNAC », 
pp. 49-87, in : Ringoot R., Utard J.-M., dirs, Le journalisme en invention. Nouvelles pratiques, nouveaux acteurs, Rennes, Presses universitaires de Rennes.

Dauvin P., 1990, « Le bulletin municipal de Rennes, souci du lecteur ou souci de l'électeur », Mots. Les langages du politique, 25, pp. 65-80.

Dauvin P., Le Bart C., 1991, « Presse municipale, presse locale », MédiasPouvoirs, 21, pp. 53-60.

Debras S., 2003, Lectrices au quotidien. Des femmes, des hommes et des journaux, Paris, Éd. L'Harmattan.

Gaxie D., 1978, Le cens caché, Paris, Éd. Le Seuil.

— 2003, « Une construction médiatique du spectacle politique ? Réalité de la contribution des médias au développement des perceptions négatives du politique », pp. 325-356, in : Lagroye J., dir., La politisation, Paris, Belin.

Goulet V., 20 I0, Médias et classes sociales. Les usages ordinaires des informations, Paris, Éd. Ina.

Hoggart R., 1957, La culture du pauvre, trad. de l'anglais par F. Garcias, J.-C. Garcias et J.-C. Passeron, Paris, Éd. de Minuit, 1970.

Katz E., Lazarsfeld P. L., 2008, Influence personnelle, trad. de l'américain par D. Cefaï, Paris,A. Colin.

Le Bart C., 1992, La rhétorique du maire entrepreneur, Paris, A. Pedone.

— 2000, « Les bulletins municipaux : une contribution ambiguë à la démocratie locale », Hermès, 26-27, pp. 175- 184.

Legavre J.-B., 2010, « Les effets paradoxaux de stratégies de communication : quelques réceptions d'un magazine territorial », communication au colloque Les effets des médias sur les électeurs, Association française de science politique, mai.

- 20II, «Entre conflit et coopération. Les journalistes et les communicants comme "associés-rivaux" 》, Communication et langages, 169, pp. I05-123.

— à paraître, « Se trouver un nom de métier. Les cas des "communicants publics" », in : Aldrin P., Hubé N., Olivier-Yaniv C., Utard J.-M., dirs, Les mondes de la communication publique, Rennes, Presses universitaires de Rennes.

Le Grignou B., 2003, Du Côté du public. Usages et réceptions de la télévision, Paris, Economica.

Leroux P., 2000, « Le bulletin municipal : de l'information militante à la presse magazine locale », pp. 6- I2, in : Leroux P., Teillet P., dirs, Le journalisme territorial. Enjeux politiques et juridiques, Paris, Centre national de la fonction publique territoriale.

Mauger G., Fossé-Poliak C., 1991, « La politique des bandes », Politix, 14, pp. 27-43.

Merton R. K., 1936, «The Unanticipated Consequences of Purposive Social Action », American Sociological Review, 6, pp. 894-904.

Rieffel R., 2005, Que sont les médias ?, Paris, Gallimard.

Saurat T., Renac L., 2009, Presse territoriale. Mode d'emploi, Paris, Territorial Éd.

Teillet P., 2000, «Tableau de la presse territoriale dans la France de l'Ouest », pp. I I3I |4, in : Leroux P., Teillet P., dirs, Le journalisme territorial, Centre national de la fonction publique territoriale. 